\title{
Boson Fock Representations of Stochastic Processes
}

\author{
L. Accardi, T. Hida, and W. W. Htay
}

UDC 519

\begin{abstract}
A classification theory of quantum stationary processes similar to the corresponding theory for classical stationary processes is presented. Our main result is the classification of those pairs of classical stationary processes that admit a joint boson Fock canonical representation.
\end{abstract}

KEY WORDS: stationary quantum stochastic process, boson Fock canonical representation, classification of quantum stochastic processes, canonical representation, canonical Markov property, multiple Markov property.

\section{Introduction}

It is known that classical probability and classical stochastic processes can be embedded in quantum probability [1]. This fact allows not only to generalize known results of classical probability theory, but also to obtain new results by looking at classical objects from a nonclassical point of view. Several papers in the last years have shown that this program can be substantiated in numerous ways in the case of classical diffusions on $\mathbb{R}^{n}[2-4]$, or on manifolds [5], and for general birth and death processes [4]. In the present note we initiate a similar program for stationary processes. The classification of these processes is not complete even in the classical case, but in the case of regular scalar-valued processes the theory is sufficiently developed [6] to admit a nontrivial quantum extension. The extension we have in mind in the present note is motivated by the analogy with quantum Brownian motion (BM): it is known that all the known boson $\mathrm{BM}$ can be realized, up to random time changes, by fixing two realizations of the classical $\mathrm{BM}$

$$
Q=(Q(s)), \quad P=(P(t)),
$$

which do not commute but have the property that their commutator is a scalar

$$
[Q(s), P(t)]=i \min \{s, t\} .
$$

Now, suppose we are given two classical stationary processes $X, Y$ which admit a canonical representation in the sense of [6] (cf. (2.1)) below) and suppose that one can realize the canonical representation of $X$ as a stochastic integral for the $Q$-process and the canonical representation of $Y$ as a stochastic integral for the $P$-process, where the stochastic integrals can be interpreted either as classical operator-valued stochastic integrals or as quantum stochastic integrals. Introducing the white noise notation, we can write

$$
Q(t)=\int_{0}^{t} q_{s} d s, \quad P(t)=\int_{0}^{t} p_{s} d s
$$

where $q_{s}$ and $p_{s}$ are white noises in the sense of [7] satisfying the commutation relation

$$
\left[q_{s}, p_{t}\right]=i \delta(s-t) \text {. }
$$

It is clear that this possibility imposes some restrictions on the pair $X, X$ and $Y$. For example, a necessary condition for this to happen is that the commutator of any pair of variables $X_{s}, Y_{t}$ is itself a scalar process of special type (see Proposition 2 below). The main result of the present note is that this necessary condition is also sufficient (see Theorem 1 below). A more delicate problem is the following: to what extent does the commutator of the two processes allow to reconstruct their canonical representation?

Translated from Matematicheskie Zametki, Vol. 67, No. 1, pp. 3-14, January, 2000.

Original article submitted August 23, 1999. 
From an analytical point of view, this problem is reduced to the problem of determining a canonical factorization of a function in the Hardy class of analytic functions. We prove that if the two stationary processes are $d$-Markov in the sense of [6] (see (4.1) below), then this problem admits a unique solution. Since the multiple Markov processes are dense in an appropriate topology in the class of stationary processes, it seems natural to conjecture that the above result holds also in the general case.

The problem we study here has natural potential applications to quantum physics. In fact, the quantum fields that are usually considered in physical models are Gaussian (but not Markovian) and have a scalar commutator. The possibility of representing these fields as stochastic integrals with respect to quantum BM would therefore put the powerful tools of classical and quantum white noise calculus directly to the service of quantum field theory, without the need to consider the stochastic limit of these fields as presently done. A complete solution of the second problem requires, however, a deeper understanding of the canonical representation for multidimensional (in fact infinite-dimensional) classical stationary processes. This problem shall be discussed elsewhere.

\section{Canonical representation of classical processes}

Definition 1. Let $X=\left\{X_{t} ; t \in[0, \infty)\right\}$ be a real-valued Gaussian process. Suppose that there exists a real-valued kernel function $F(t, u)$ such that the process $Y=\left\{Y_{t} ; t \in[0, \infty)\right\}$ given by

$$
Y_{t}=\int_{0}^{t} F(t, u) d B(u)
$$

satisfies $B_{t}(Y)=B_{t}(B)$ for every $t$, where $B_{t}(Y)$ and $B_{t}(B)$ are the $\sigma$-fields generated by $Y_{s}$ and $B(s), \quad s \leq t$, respectively, and has the same finite-dimensional joint distributions as $X$ (in this case? following a widespread probabilistic terminology, $Y$ is called a version of the process $X_{t}$ ). In this case the pair $\{F(t, u), u \leq t ; B(t)\}$ (simply written as $\{F(t, u), B\}$ ) is called a canonical representation of $X$, and $F(t, u)$ is a canonical kernel.

Sometimes in the existing literature, the term canonical representation is used for the stronger condition that $X_{t}$ itself is expressed as in (2.1), i.e., $Y_{t}=X_{t}$. In this case the Brownian motion $B$ above is the innovation process of $X$. If $X_{t}$ is stationary, one can choose $F(t, u)=F(t-u)$.

Definition 2. Two classical stationary processes $\tilde{X}, \bar{Y}$ are said to admit a joint representation if their canonical representations can be realized on the same probability space.

In other words, $\tilde{X}$ and $\tilde{Y}$ admit a joint representation, if there exist versions (in the sense specified above) $X$ and $Y$ of $\widetilde{X}$ and $\widetilde{Y}$ respectively, where the canonical representations are expressed in the forms

$$
X_{t}=\int G(t-u) W(u) d u \quad Y_{t}=\int F(t-u) W(u) d u
$$

by using the same white noise $\{W(u)\}$. The kernels $G(u)$ and $F(u)$ vanish for $u<0$.

Notice that for scalar-valued processes admitting a canonical representation, a joint representation can always be constructed, so Definition 2 above is nontrivial only in the case of multidimensional processes.

In the present note we shall freely use the terminology of quantum probability. The notion of stochastic process we use is the same as in [1]. The quantum white noise (1.4) is realized on the Fock space over $L^{2}(\mathbb{R})$ corresponding to the fact that we consider only quantum analogs of scalar-valued processes which are realized as operators on this Fock space. In particular by a classical process we mean a family $\left(X_{t}\right)(t \in \mathbb{R})$ of self-adjoint operators on the above defined Fock space such that the exponentials $\exp \sum_{j=1}^{n} a_{j} X_{t_{j}}$ are well defined for any finite family of real numbers $t_{1}, \ldots, t_{n}, a_{1}, \ldots, a_{n}$ and the family of these exponentials generates an Abelian von Neumann algebra. The term stationary for such processes shall always refer to their distributions with respect to the vacuum vector $\Phi$, so that the stationarity condition means that

$$
\left\langle\Phi, \exp \sum_{j=1}^{n} a_{j} X_{t_{j}+s} \Phi\right\rangle=\left\langle\Phi, \exp \sum_{j=1}^{n} a_{j} X_{t_{j}} \Phi\right\rangle
$$

for any finite family of real numbers $t_{1}, \ldots, t_{n}, a_{1}, \ldots, a_{n}$ for any real number $s$. 
Definition 3. Two classical stationary processes $X, Y$ admit a joint boson Fock representation if there exists a boson Fock white noise

$$
\Gamma\left(\mathcal{H}_{1}\right)=:\left\{\mathcal{H}, \Phi, q_{t}, p_{t}\right\}
$$

such that $X$ and $Y$ can be respectively expressed (up to isomorphism in the sense of finite-dimensional joint distributions) in the in the form

$$
X_{t}=\int G(t-u) q_{u} d u, \quad Y_{t}=\int F(t-u) p_{u} d u
$$

for two real-valued functions $F$ and $G$. The representation (2.4) is called canonical if the representation of $X$ is canonical for the $q$-white noise and that of $Y$ is canonical for the $p$-white noise. Notice that, since the processes are classical, it makes sense to speak of their canonical representations in the sense of Definition 1.

Proposition 1. If two classical stationary processes $X, Y$ admit a joint Fock representation if and only if they admit a joint representation in the classical sense.

Proof. Recall that we identify the classical white noise with the $p$-process in Fock space and let $\Gamma(i)$ denote the Fourier-Gauss transform. Then

$$
q_{u}=\Gamma(i) p_{u} \Gamma(i)^{*}, \quad u \in \mathbb{R}
$$

The process $\tilde{Y}_{t}=\Gamma(i) Y_{t} \Gamma(i)^{*}$ has the same vacuum distribution as $Y_{t}$ because $\Gamma(i) \Phi=\Phi$. If $Y_{t}$ has the form (2.4) then $\tilde{Y}_{t}$ shall have the form

$$
\widetilde{Y}_{t}=\int F(t-u) q_{u} d u
$$

Since a unitary isomorphism transforms the conditional expectations of the $Y$-process into the corresponding ones of the $\widetilde{Y}$-process, it follows that, if the representation of $Y$ is canonical, then (2.5) is isomorphic to the canonical representation of $\bar{Y}$. From this remark the statement follows immediately because now the two processes $X$ and $\tilde{Y}$ are implemented in the same von Neumann Abelian algebra; hence they are given on the same probability space with the same filtration.

The following is a well-known and elementary fact:

Lemma 1. The space of integrable functions vanishing on the negative half-line is an algebra under convolution.

Proof. Let $F(t)$ and $G(t)$ be integrable functions vanishing for $t<0$. Then their convolution is well defined and it is integrable [8]. By inspection of the identity

$$
F * G(t)=\int_{-\infty}^{t} F(t-s) G(s) d s
$$

one immediately sees that $F * G$ is again vanishing on the negative half-line.

Remark 1. A corollary to the above lemma is that the space of Fourier transforms of integrable functions vanishing on the negative half-line forms an algebra under pointwise multiplication. In the following we shall always denote by $\widehat{F}$ the Fourier transform of the function $F$. 
Proposition 2. If two quantum stationary processes $X$ and $Y$ admit a joint boson Fock representation then, in this representation, for each real numbers $s, t$ the domains of $X_{s}$ and $Y_{t}$ have a dense intersection and their commutator has the form

$$
\left[X_{s}, Y_{t}\right]=i \Theta(s-t)=i \int e^{-i(s-t) \lambda} \hat{\Theta}(\lambda) d \lambda, \quad s, t \in \mathbb{R}
$$

where $\widehat{\Theta}$ is the Fourier transform of a function which vanishes on the negative half line and the commutator is understood weakly on the intersection of the domains. Moreover, the function $\Theta$ is uniquely determined by the identity

$$
\widehat{\Theta}(\lambda)=\widehat{G}(\lambda) \overline{\widehat{F}(\lambda)},
$$

where $F$ and $G$ are defined by Eq. (2.2).

Proof. Let a representation of $X$ and $Y$ be realized in the form (2.4). Then, let us introduce the spectral representation of the white noises $q$ and $p$

$$
q_{\lambda}=\frac{1}{\sqrt{2 \pi}} \int e^{i t \lambda} q_{t} d t, \quad p_{\lambda}=\frac{1}{\sqrt{2 \pi}} \int e^{-i t \lambda} p_{t} d t
$$

Since $F$ and $G$ are real, we can write their commutator in the form

$$
\left[X_{s}, Y_{t}\right]=i \int_{-\infty}^{t \wedge s} G(s-u) F(t-u) d u=i \int_{-\infty}^{\infty} e^{-i(s-t) \lambda} \widehat{G}(\lambda) \overline{\widehat{F}(\lambda)} d \lambda
$$

The function $\widehat{\Theta}(\lambda):=\widehat{G}(\lambda) \overline{\widehat{F}(\lambda)}$ is the Fourier transform of $(G * F)(t)$, which vanishes on the negative half-line.

Definition 4. A joint Hilbert space representation of two classical stationary processes $X, Y$ is a pair $\{\mathcal{H}, \Phi\}$, where $\mathcal{H}$ is a Hilbert space and where $\Phi$ is a unit vector in $\mathcal{H}$ such that for each $t \in R, X_{t}$ and $Y_{t}$ are represented as operators on $\mathcal{H}$ (still denoted by $X_{t}, Y_{t}$ for simplicity).

Definition 5. Two Hilbert space representations $\{\mathcal{H}, \Phi\},\left\{\mathcal{H}^{\prime}, \Phi^{\prime}\right\}$ are said to be equivalent if there exists a unitary isomorphism $U: \mathcal{H} \rightarrow \mathcal{H}^{\prime}$ such that $U \Phi=\Phi^{\prime}$ and that $U$ intertwines the actions of $X$ and $Y$ on $\mathcal{H}$ and $\mathcal{H}^{\prime}$, respectively.

Remark 2. From this definition it is clear that any pair of stochastic processes has a joint Hilbert space representation. It is sufficient, to this end, to consider the standard Hilbert space representations $\left\{\mathcal{H}_{X}, \Phi_{X}\right\},\left\{\mathcal{H}_{Y}, \Phi_{Y}\right\}$ of $X$ and $Y$ defined by Lemma 1 and to form their tensor product

$$
\mathcal{H}:=\mathcal{H}_{X} \otimes \mathcal{H}_{Y} ; \quad \Phi:=\Phi_{X} \otimes \Phi_{Y}
$$

However it is clear that, if the processes are classical, this representation in general will not be the canonical one.

We want to study the following problems:

Given a joint Hilbert space representation $\{\mathcal{H}, \Phi\}$ of two classical stationary processes $X$ and $Y$. Under what conditions is this representation equivalent to a boson Fock representation of $X$ and $Y$ ?

Proposition 1 and Proposition 2 give two necessary conditions for this to be the case. Namely,

i) $X, Y$ must have a canonical representation;

ii) the commutator of $X$ and $Y$ should be well defined, weakly on a dense domain and on this domain it should have the form (2.6), (2.9). 


\section{Sufficiency of the condition}

Let $X=\left(X_{t}\right)$ and $Y=\left(Y_{t}\right)$ be stationary classical stochastic processes realized as operators on a Hilbert space $\mathcal{H}$ with a unit vector $\Phi$ such that

$$
\begin{aligned}
\left\langle\Phi, f\left(\left\{X_{t}\right\}\right) \Phi\right\rangle & =E\left(f\left(\left\{X_{t}\right\}\right)\right), \\
\left\langle\Phi, f\left(\left\{Y_{t}\right\}\right) \Phi\right\rangle & =E\left(f\left(\left\{Y_{t}\right\}\right)\right),
\end{aligned}
$$

where $f\left(\left\{X_{t}\right\}\right)$ denotes an arbitrary functional of the process $X_{t}$ and similarly for $Y_{t}$. Notice that the left-hand side of Eqs. (3.1), (3.2) is well defined by the spectral theorem for any Borel function $f$ on $\mathbb{R}$ depending only on a finite number of random variables. This identification can be extended to arbitrary measurable functionals using the canonical identification of the Abelian algebra generated by the random variables with the algebra of bounded functionals of the classical process.

Suppose, moreover, that $X$ and $Y$ have a joint representation $\{\mathcal{H}, \Phi\}$ in the sense of Definition 4 :

$$
\begin{aligned}
& X_{t}=\int e^{-i t \lambda} \widehat{G}(\lambda) q_{\lambda} d \lambda, \\
& Y_{t}=\int e^{i t \lambda} \overline{\widehat{F}(\lambda)} p_{\lambda} d \lambda,
\end{aligned}
$$

where $q_{\lambda}$ and $p_{\lambda}$ are standard white noises with respect to the vector $\Phi$ and, further, each of the representations (3.3) is isomorphic to the canonical representation of the corresponding classical process. Suppose also that, for introducing the processes

$$
\frac{1}{\sqrt{2}}\left(q_{\lambda}+i p_{\lambda}\right)=a_{\lambda}, \quad \frac{1}{\sqrt{2}}\left(q_{\lambda}-i p_{\lambda}\right)=a_{\lambda}^{+}
$$

The vacuum vector $\Phi$ is in the domain of the polynomial algebra generated by $a_{\lambda}^{ \pm}$(in the sense of distributions) and

$$
a_{\lambda} \Phi=0 \text {. }
$$

Notice that here we are not assuming any commutation relation between the two white noises $p$ and $q$. Our goal is to deduce these commutation relations from the commutation relations of the processes $X$ and $Y$. This will prove that the necessary condition considered in the previous section is also sufficient.

Lemma 2. Let $M, N$ be operator-valued measures on $\mathbb{R} \times \mathbb{R}$ such that for any $u \in \mathbb{R}^{2}$ one has

$$
\int_{\mathbb{R}^{2}} e^{i u \cdot \alpha} M(d \alpha)=\int_{\mathbb{R}^{2}} e^{i u \cdot \alpha} N(d \alpha)
$$

where $u \cdot \alpha$ is the scalar product in $\mathbb{R}^{2}$. Then $M=N$.

Proof. This follows by taking matrix elements of the identity (3.6) and using the uniqueness of the Fourier transform of complex measures.

Now let us assume that condition (2.6) is satisfied for some function $\Theta$ satisfying the conditions of Proposition 2. Then, explicit calculation of the commutator of $X$ and $Y$ using (3.3) gives:

$$
\begin{aligned}
{\left[X_{s}, Y_{t}\right] } & =\iint e^{-i s \lambda} e^{i t \lambda^{\prime}} \widehat{G}(\lambda) \overline{\widehat{F}\left(\lambda^{\prime}\right)}\left[q_{\lambda}, p_{\lambda^{\prime}}\right] d \lambda d \lambda^{\prime} \\
& =i \int e^{-i(s-t) \lambda} \widehat{\Theta}(\lambda) d \lambda=i \iint e^{-i s \lambda} e^{i t \lambda^{\prime}} \widehat{\Theta}(\lambda) \delta\left(\lambda-\lambda^{\prime}\right) d \lambda d \lambda^{\prime}
\end{aligned}
$$

from Lemma (3.1), it follows that, in the sense of distributions

$$
\widehat{G}(\lambda) \overline{\widehat{F}\left(\lambda^{\prime}\right)}\left[q_{\lambda}, p_{\lambda^{\prime}}\right]=i \widehat{\Theta}(\lambda) \delta\left(\lambda-\lambda^{\prime}\right)
$$


In the identity (3.8), if the product $\widehat{G}(\lambda) \overline{\widehat{F}\left(\lambda^{\prime}\right)}$ is zero on a set of zero Lebesgue measure, then we can change this value without altering the identity (3.8). If it is zero on a set of strictly positive Lebesgue measure on the diagonal $\lambda=\lambda^{\prime}$, then also $\hat{\theta}(\lambda)$ must be zero. Therefore, with the convention that the quotient below is zero at those points on the diagonal at which the denominator vanishes, we can write:

$$
\left[q_{\lambda}, p_{\lambda^{\prime}}\right]=i \frac{\widehat{\Theta}(\lambda)}{\widehat{G}(\lambda) \hat{\widehat{F}\left(\lambda^{\prime}\right)}} \delta\left(\lambda-\lambda^{\prime}\right)
$$

Now, starting from the white noises $q_{\lambda}, p_{\lambda^{\prime}}$ in (3.3), let us define the processes $q_{t}$ and $p_{t}$ by the relation (2.8). Then one has

$$
\left[q_{\lambda}, p_{\lambda^{\prime}}\right]=\frac{1}{2 \pi} \iint e^{i s \lambda} e^{-i t \lambda^{\prime}}\left[q_{s}, p_{t}\right] d s d t .
$$

and therefore, taking the inverse Fourier transform of (3.9), we find:

$$
\left[q_{s}, p_{t}\right]=\frac{1}{2 \pi} i \iint e^{-i s \lambda} e^{i t \lambda^{\prime}} \frac{\widehat{\Theta}(\lambda)}{\widehat{\widehat{G}(\lambda) \widehat{F}\left(\lambda^{\prime}\right)}} \delta\left(\lambda-\lambda^{\prime}\right) d \lambda d \lambda^{\prime}=\frac{1}{2 \pi} i \int e^{-i(s-t) \lambda} \frac{\widehat{\Theta}(\lambda)}{\widehat{G}(\lambda) \widehat{\hat{F}}(\lambda)} d \lambda .
$$

From these relations, it follows that

$$
\frac{1}{2 \pi} \int e^{-i(s-t) \lambda} \frac{\widehat{\Theta}(\lambda)}{\widehat{G}(\lambda) \widehat{\widehat{F}(\lambda)}} d \lambda=g(s-t)
$$

for some function or distribution $g(s-t)$. We want to prove that $g(s-t)=\delta(s-t)$.

Lemma 3. Let a Hilbert space $\mathcal{H}$ and a unit vector $\Phi$ in it be given, and let two self-adjoint operators on $\mathcal{H}, q, p$, which have standard Gaussian $\Phi$-distributions (cf. Definition 4) be given. Suppose, moreover, that $[q, p]=i c, c \neq 0, c \in \mathbb{R}$, and that one can exponentiate this relation into the Weyl relations

$$
e^{i s p} e^{i t q}=e^{-i s t} e^{i t q} e^{i s p}
$$

Then $c=1$.

Proof. Suppose the contrary. Possibly exchanging the roles of $p$ and $q$, we can assume that $c>0$. The new pair

$$
P:=\frac{p}{\sqrt{c}}, \quad Q:=\frac{q}{\sqrt{c}}
$$

then satisfies $[Q, P]=i$. The assumption on the possibility of exponentiating the commutation relations between $q$ and $p$ implies that the system $\{\mathcal{H}, \Phi, Q, P\}$ is isomorphic, up to multiplicity of the representation, to the Schrödinger representation. This implies that $Q, P$ are $\Phi$-standard Gaussian. Therefore,

$$
\left\langle\Phi, e^{i q \lambda} \Phi\right\rangle=\left\langle\Phi, e^{i \sqrt{c} \lambda Q} \Phi\right\rangle=\int e^{i \sqrt{c} x \lambda} \frac{e^{-\frac{x^{2}}{2}}}{(2 \pi)^{\frac{1}{2}}} d x=\int e^{i y \lambda} \frac{e^{-\frac{y^{2}}{2 c}}}{(2 \pi c)^{\frac{1}{2}}} d y .
$$

So $q$ is $\Phi$-Gaussian with mean zero and variance $c$. But, according to our assumption $q$, is standard Gaussian, so $c=1$. In the notations above, let us apply this result to the case in which

$$
\left[q_{s}, p_{t}\right]=i g(s-t) \text {. }
$$

Introducing test functions $\varphi$ and $\psi$ and the association smeared noises, we have

$$
q(\varphi)=\int \varphi(s) q_{s} d s, \quad p(\psi)=\int \psi(s) p_{s} d s
$$

Eq.(3.10) is equivalent to

$$
[q(\varphi), p(\psi)]=i \iint \varphi(s) g(s-t) \psi(t) d s d t
$$


Theorem 1. Let $X$ and $Y$ be as in (3.3) with $q_{\lambda}, p_{\lambda}$ satisfying (3.5) and the conditions specified after formula (3.3). Suppose there exists a scalar integrable function $\widehat{\Theta}$ such that the commutator of $X$ and $Y$ has the form

$$
\left[X_{s}, Y_{t}\right]=i \int e^{-i(s-t) \lambda} \widehat{\Theta}(\lambda) d \lambda,
$$

and suppose that for any pair of test functions $\varphi, \psi$, the operators $q(\varphi)$ and $p(\psi)$ defined by (3.10) satisfy the conditions of Lemma 3. Then the white noises $q_{\lambda}, p_{\lambda}$ are the spectral representations of a boson Fock white noise $q_{t}, p_{t}$.

Proof. By polarization, the commutator $[q(\varphi), p(\psi)]$ is uniquely determined in the case $\varphi=\psi$. Fix $\varphi$ and suppose that

$$
\int \varphi^{2}(s) d s=1
$$

Then by Lemma $3, q(\varphi), p(\varphi)$ are standard $\Phi$-Gaussian and

$$
[q(\varphi), p(\psi)]=i \iint \varphi(s) g(s-t) \psi(t) d s d t
$$

By Lemma 3, we prove that (3.12) implies

$$
\iint \varphi(s) g(s-t) \varphi(t) d s d t=1
$$

By the polarization identity, one can prove that Eqs. (3.12) and (3.13) are equivalent to

for any $\varphi$ and $\psi$. This implies

$$
\iint \varphi(s) g(s-t) \psi(t) d s d t=\iint \varphi(s) \delta(s-t) \psi(t) d s d t
$$

$$
g(s-t)=\delta(s-t)
$$

But by our assumption and (2.8), the processes (3.4) annihilates the vacuum. This, together with the cormmutation relation (3.10), is sufficient to guarantee that the pair $q_{t}, p_{t}$ is isomorphic to the standard Boson Fock white noise.

\section{Canonical property and multiple Markov property}

Before we come to the next question, we give a short review of the canonical representation of a classical Gaussian process.

Suppose $X_{t}$ is given by (2.2). Then, it is known that the Fourier transform $\widehat{G}(\lambda)$ of $G$ is in the Hardy class (see [9]) and therefore is the boundary value of an analytic function $\widehat{G}(w)$ in the lower half-plane $C^{-}$.

Theorem 2. Let $X, Y$ be classical stochastic processes, $\{\mathcal{H}, \Phi\}$ a Hilbert space representation of the pair $X, Y$. Suppose that the conditions of Theorem 1 are satisfied. Then, denoting by

$$
\widehat{\Theta}=C_{\Theta} B_{\Theta} S_{\Theta}, \quad \widehat{G}=C_{1} B_{1} S_{1}, \quad \widehat{F}=C_{2} B_{2} S_{2}
$$

the canonical decompositions of $\widehat{\Theta}, \widehat{G}$ and $\widehat{F}$ respectively, we see that $\{\mathcal{H}, \Phi\}$ is isomorphic, in the sense of Definition 5 to the boson Fock representation of $X, Y$ if and only if

$$
C_{\Theta}=C_{1} \bar{C}_{2} ; \quad B_{\Theta}=B_{1} \bar{B}_{2} ; \quad S_{\Theta}=S_{1} \bar{S}_{2}
$$

Proof. This follows from the uniqueness of the decomposition of Hardy functions.

Now assume that $X_{t}$ is a multiple Markov process of order $N<+\infty$. This is a rather natural assumption for $X_{t}$ to be a mathematical model of some physical phenomenon. For the definition of the multiple Markov property of a (classical) Gaussian process, we refer to [6, Chap. 5].

This definition is equivalent to the fact that $\widehat{G}(\lambda)$ is expressed as a rational function of the form

$$
C(\lambda)=\frac{Q(i \lambda)}{P(i \lambda)}, \quad-\infty<\lambda<\infty,
$$

where $P$ and $Q$ are polynomials with degree of $Q<$ degree of $P=N$ and they have no roots with positive real part (see $[6$, Sec. 5.4]). 


\section{Factorization}

Before we come to the factorization of $\widehat{\Theta}$, we extend to noncommuting pairs of the classical stochastic processes the notion of multiple Markov property.

Let $X_{t}$ and $Y_{t}$ be classical stochastic processes given by

$$
X_{t}=\int^{t} G(t, u) q_{u} d u, \quad Y_{t}=\int^{t} F(t, u) p_{u} d u
$$

where $G$ and $F$ are canonical kernels.

Definition 6. The quantum stochastic process defined by the pair $X$ and $Y$ is said to be multiple Markov if the vacuum distributions of $X_{t}$ and $Y_{t}$ respectively define multiple Gauss-Markov processes in the classical sense.

Coming to the stationary quantum stochastic processes $X_{t}$ and $Y_{t}$ given by (2.4), we assume that they are multiple Markov. Then, by [6, Chap. 5] again, the canonical kernels $G$ and $F$ are of Goursat type. This means that their Fourier transforms are expressed as rational functions in the form (4.1), say

$$
\widehat{G}(\lambda)=\frac{Q_{1}(i \lambda)}{P_{1}(i \lambda)}, \quad \widehat{F}(\lambda)=\frac{Q_{2}(i \lambda)}{P_{2}(i \lambda)},
$$

where $P_{i}, Q_{i}, i=1,2$ are all polynomials. We shall assume, in addition, that they have no zeros on the real line.

With this background, we now come to the factorization problem of $\widehat{\Theta}(\lambda)$.

By assumption $\widehat{\Theta}(\lambda)$ is given as in (2.7). Notice that the asymmetric role of $X$ and $Y$ in formula (2.7) has its role in the Definition 4 of spectral transform of quantum white noise, and this is motivated by the necessity of preserving the commutation relations.

Hence, we must have

$$
\hat{\Theta}(\lambda)=\frac{Q_{1}(i \lambda) \overline{Q_{2}(i \lambda)}}{P_{1}(i \lambda) \overline{P_{2}(i \lambda)}} .
$$

Our problem is now reduced to determine $P_{i}$ and $Q_{i}, \quad i=1,2$. The given function $\widehat{\Theta}$ should be a product of two functions, say $\widehat{\Theta}_{1}(\lambda)$ and $\widehat{\widehat{\Theta}}_{2}(\lambda)$, that are the boundary values of $\widehat{\Theta}_{1}(w) \widehat{\widehat{\Theta}}_{2}(w)$, and the latter are rational functions of $w$ and $\bar{w}$, respectively. Take the poles of $\widehat{\Theta}$ in $\mathbb{C}^{+}$. They must be zero points of $P_{1}$. The zero points of $\widehat{\Theta}$ in $\mathbb{C}^{+}$must be the zero points of $Q_{1}$. Similarly the poles and the zeros in $\mathbb{C}^{-}$must be the zero points of $\bar{P}_{2}$ and $\bar{Q}_{2}$ respectively.

Thus, these $P_{i}, Q_{i}, i=1,2$ are determined up to constants. This means that $\widehat{G}$ and $\widehat{F}$ are determined up to constants.

It should be noted that the degree of $P_{1}$ and the degree of $P_{2}$ are the multiplicities in the Markov property for $X_{t}$ and $Y_{t}$, respectively.

Theorem 3: Assume that two quantum stochastic processes $X_{t}$ and $Y_{t}$ are multiple Markov and satisfy the conditions of Theorem 1. Then, given the commutator of $X_{t}$ and $Y_{t}$, the orders of the Markov property of $X_{t}$ and $Y_{t}$ are uniquely determined, and the canonical representations of $X_{t}$ and $Y_{t}$ of the form (2.4) are uniquely determined up to constant factors.

\section{Concluding remarks}

We would like to conclude with some very preliminary comments on the case when noncanonical representations are involved.

In these cases there are more possibilities of factoring the function $\widehat{\Theta}(\lambda)$, defining the commutator of the two processes. For example, if we still assume that $X, Y$ are multiple Gauss-Markov processes and, moreover, that there is no singular factor in $\widehat{\Theta}, \widehat{G}$ and $\widehat{F}$, then we may write $\widehat{G}(\lambda)=C_{1}(\lambda) B_{1}(\lambda)$, 
$\widehat{F}(\lambda)=C_{2}(\lambda) B_{2}(\lambda)$, where $C_{i}$ are rational functions corresponding to the canonical kernel and $B_{i}$ is the Blaschke product, $i=1,2$. In this case $\widehat{\Theta}(\lambda)=C_{1}(\lambda) \overline{C_{2}(\lambda)} \cdot B_{1}(\lambda) \overline{B_{2}(\lambda)}$, and it is known that

$C_{1}(\lambda)$ has no zero and no pole in the lower half plane $\mathbb{C}^{-}$.

$\overline{C_{2}(\lambda)}$ has no zero and no pole in the upper half plane $\mathbb{C}^{+}$.

$B_{1}(\lambda)$ has zeros in $\mathbb{C}^{-}$and poles in $\mathbb{C}^{+}$.

$\overline{B_{2}(\lambda)}$ has zeros in $\mathbb{C}^{+}$and poles in $\mathbb{C}^{-}$.

If we take the poles of $\widehat{\Theta}$ in $\mathbb{C}^{+}$as in the canonical case, these poles may be either those of $C_{1}$ or of $B_{1}$. A similar ambiguity can be seen for the zeros and poles in $\mathbb{C}^{-}$.

We conjecture that, by introducing some optimality conditions on $|\hat{\Theta}(w)|$ along the same lines as those discussed in [10], it should be possible to individualize the contributions of the Blaschke products to the poles and the zeros. However, this problem will be discussed elsewhere.

The authors express their gratitude to the referee for a careful reading of the present paper and for detailed remarks that allowed to improve its clarity.

\section{References}

1. L. Accardi and F. Fagnola, "Quaegebeur quantum stochastic calculus," J. Funct. Anal., 104, 149-197 (1992); Volterra preprint, No. 18, (1990).

2. B. V. R. Bhat, F. Fagnola, and B. Sinha, "On quantum extensions of semigroups of Brownian motion on a half-line," Russian J. of Math. Phys., 4, No. 3, 13-28 (1996).

3. A. M. Chebotarev, "Minimal solution in classical and quantum stochastics," in: Quantum Probability and Related Topics, Vol. 7, World Scientific, Singapore (1992), pp. 79-97.

4. F. Fagnola and R. Monte, "On quantum extension of the semigroup of Bessel process quantum extensions of semigroups," Math. Notes, 60, No. 4, 519-537 (1996).

5. L. Accardi and A. Mohari, "On the structure of classical and quantum flows," Volterra Preprint, No. 167 (1994).

6. T. Hida and M. Hitsuda, "Gaussian Processes," Amer. Math. Soc., Providence, R. I. (1993).

7. T. Hida, Brownian Motion, Springer-Verlag, New York (1980).

8. A. N. Kolmogorov and S. V. Fomin, Introductory Real Analysis, Dover Publications, New York (1970).

9. K. Hoffman, Banach Spaces of Analytic Functions., Prentice-Hall (1962).

10. W. W. Htay, "Optimalities for random functions: Lee-Wiener's network and non-canonical representation of stationary Gaussian processes," Nagoya Math. J., 149, 9-17 (1998).

(L. Accardi) Graduate School of Polymathematics, Nagoya University AND ROMA UNIVERSITY TOR VERGata

E-mail address: accardi@volterra.mat.utovrm.it

(T. Hida) Faculty of Science and Technology, Meijo University

E-mail address: thida@meijo-u.ac.jp

(W. W. Htay) Faculty of Science and Technology, Meijo University 